\title{
An initial value problem for a third order differential equation
}

\author{
by Wojciech MydlarczyK (Wrocław)
}

Abstract. For an initial value problem $u^{\prime \prime \prime}(x)=g(u(x)), u(0)=u^{\prime}(0)=u^{\prime \prime}(0)=0$, $x>0$, some theorems on existence and uniqueness of solutions are established.

1. Introduction. We consider the initial value problem

$$
\begin{aligned}
& u^{\prime \prime \prime}(x)=g(u(x)) \quad(x>0), \\
& u(0)=u^{\prime}(0)=u^{\prime \prime}(0)=0,
\end{aligned}
$$

where

(i) $g:(0, \infty) \rightarrow[0, \infty)$ is continuous,

(ii) $x^{1 / 2} g(x)$ is bounded as $x \rightarrow 0$,

(iii) $0<\int_{0}^{\delta} g(s) s^{-1 / 2} d s<\infty(\delta>0)$.

In the paper $\delta$ always denotes some positive constant. We permit it to change its value from paragraph to paragraph.

We are looking for a nonnegative function $u \in C^{2}[0, \delta)$ satisfying (1.1). It is known (see [1]-[3]) that for $g$ nondecreasing a similar problem for the $n$th order differential equation

$$
\begin{aligned}
& u^{(n)}(x)=g(u(x)), \\
& u(0)=u^{\prime}(0)=\ldots=u^{(n-1)}(0)=0 \quad(n \text { an integer }),
\end{aligned}
$$

has a nontrivial solution $u \not \equiv 0$ if and only if the generalized Osgood condition

$$
\int_{0}^{\delta}\left(\frac{s}{g(s)}\right)^{1 / n} \frac{d s}{s}<\infty \quad(\delta>0)
$$

is satisfied. For further results of this type, see [4], [5].

1991 Mathematics Subject Classification: 45D05, 45G10.

Key words and phrases: nonlinear Volterra integral equation, existence and uniqueness of solutions. 
Our aim is to give a similar condition for the existence of nontrivial solutions of (1.1) in the case of a class of functions $g$ satisfying (i)-(iii).

To study the problem (1.1) we transform it into its integral form

$$
u(x)=\frac{1}{2} \int_{0}^{x}(x-s)^{2} g(u(s)) d s \quad(0<x<\delta) .
$$

Taking $v(x)=2^{-2 / 3}\left[u^{\prime}\left(u^{-1}(x)\right)\right]^{2}$, where $u^{-1}$ is the inverse function to $u$, we get the integral equation

$$
v(x)=\int_{0}^{x}(x-s) g(s) v(s)^{-1 / 2} d s \quad(0<x<\delta) .
$$

The integral equation (1.2), with an unknown nonnegative function $v \in$ $C[0, \delta)$, will be equivalent to problem (1.1) if we assume

$$
\int_{0}^{\delta} v(s)^{-1 / 2} d s<\infty \quad(\delta>0)
$$

which is motivated by the relation $v(x)^{-1 / 2}=2^{1 / 3}\left(u^{-1}\right)^{\prime}(x)$.

To analyse (1.2), we introduce the integral operator

$$
T w(x)=\int_{0}^{x}(x-s) g(s) w(s)^{-1 / 2} d s \quad(x>0)
$$

defined for any continuous function $w:[0, \infty) \rightarrow[0, \infty)$ such that the integral on the right-hand side is finite, and set

$$
\varphi(x)=\int_{0}^{x}(x-s) g(s) s^{-1 / 2} d s \quad \text { and } \quad q(x)=x^{1 / 3} \varphi(x)^{2 / 3} \quad(x>0) .
$$

Some properties of $\varphi$ and $q$ which we shall use in the sequel are collected in the two following lemmas.

Lemma 1.1. Let $g$ satisfy (i)-(iii). Then

(a) $\varphi(x) / x$ is nondecreasing for $x>0$;

(b) there exists a constant $c>0$ such that $x \varphi^{\prime 2}(x) \leq c \varphi(x)$ for $x \in(0, \delta)$;

(c) if $x^{1 / 2} g(x)$ is nondecreasing for $x \in(0, \delta)$, then $x \varphi^{\prime 2}(x) / \varphi(x)$ is also nondecreasing on $(0, \delta)$.

Proof. (a) It suffices to observe that $\varphi$ is convex and $\varphi(0)=0$.

(b) Notice that

$$
x \varphi^{\prime 2}(x)=\int_{0}^{x}\left(2 s \varphi^{\prime \prime}(s)+\varphi^{\prime}(s)\right) \varphi^{\prime}(s) d s \quad(x>0) .
$$

Now, our assertion follows by the fact that $2 s \varphi^{\prime \prime}(s)+\varphi^{\prime}(s)$ is bounded on $(0, \delta)$. 
(c) We first note that

$$
\varphi^{2}\left(x \varphi^{\prime 2} / \varphi\right)^{\prime}=\left\{\left(2 x \varphi^{\prime \prime}+\varphi^{\prime}\right) \varphi-x \varphi^{\prime 2}\right\} \varphi^{\prime} .
$$

Further, from (1.5) and by the fact that $2 s \varphi^{\prime \prime}(s)+\varphi^{\prime}(s)$ is nondecreasing on $(0, \delta)$ we obtain

$$
x \varphi^{\prime 2}(x) \leq\left(2 x \varphi^{\prime \prime}(x)+\varphi^{\prime}(x)\right) \varphi(x) \text { for } x \in(0, \delta) .
$$

So, $\left(x \varphi^{\prime 2} / \varphi\right)^{\prime} \geq 0$ on $(0, \delta)$, which completes the proof.

LEMma 1.2. Let $g$ satisfy (i)-(iii). Then

(a) $T q(x)$ is well defined for $x>0$;

(b) if $x^{1 / 2} g(x)$ is nondecreasing on $(0, \delta)$, then $T q(x) \leq \frac{9}{2} q(x)$ for $x \in$ $(0, \delta)$.

Proof. (a) We first observe that

$$
\int_{0}^{x} g(s) q(s)^{-1 / 2} d s=\int_{0}^{x} \varphi^{\prime \prime}(s)\left(\frac{\varphi(s)}{s}\right)^{-1 / 3} d s \quad(0<x) .
$$

By the estimate given in Lemma 1.1(b) we can integrate by parts to obtain

$$
\begin{aligned}
\int_{0}^{x} \varphi^{\prime \prime}(s)\left(\frac{\varphi(s)}{s}\right)^{-1 / 3} d s & =\varphi^{\prime}(x)\left(\frac{\varphi(x)}{x}\right)^{-1 / 3} \\
& +\frac{1}{3} \int_{0}^{x} \varphi^{\prime}(s)\left(\frac{\varphi(s)}{s}\right)^{-1 / 2}\left(\frac{\varphi(s)}{s}\right)^{\prime}\left(\frac{\varphi(s)}{s}\right)^{-5 / 6} d s .
\end{aligned}
$$

In order to estimate the right-hand side of (1.6) we use Lemma 1.1(b) once more, which gives

$$
\int_{0}^{x} \varphi^{\prime \prime}(s)\left(\frac{\varphi(s)}{s}\right)^{-1 / 3} d s<\infty \quad \text { for } x \in(0, \delta) .
$$

Hence our assertion follows immediately.

(b) We use Lemma 1.1(c) in order to estimate the integral on the righthand side of (1.6). Then we obtain

$$
\int_{0}^{x} \varphi^{\prime \prime}(s)\left(\frac{\varphi(s)}{s}\right)^{-1 / 3} d s \leq 3 \varphi^{\prime}(x)\left(\frac{\varphi(s)}{s}\right)^{-1 / 3} \quad \text { for } x \in(0, \delta) .
$$

Now, we derive

$$
\begin{aligned}
T q(x) & =\int_{0}^{x}\left(\int_{0}^{s} g(\xi) q(\xi)^{-1 / 2} d \xi\right) d s \\
& =3 \int_{0}^{x} \varphi^{\prime}(s)\left(\frac{\varphi(s)}{s}\right)^{-1 / 3} d s \leq \frac{9}{2} q(x) \quad \text { for } x \in(0, \delta),
\end{aligned}
$$

which completes the proof. 
In view of Lemma 1.2(a), we can put

$$
Q(x)=T q(x) \quad \text { for } x \in(0, \delta) .
$$

In the following theorem necessary and sufficient conditions for the existence of nontrivial solutions $u \not \equiv 0$ of (1.1) are established.

Theorem 1.1. Let (i)-(iii) be satisfied. If

$$
\int_{0}^{\delta} q(s)^{-1 / 2} d s<\infty \quad(\delta>0)
$$

then (1.1) has a nontrivial solution. Conversely, if (1.1) has a nontrivial solution, then

$$
\int_{0}^{\delta} Q(s)^{-1 / 2} d s<\infty \quad(\delta>0)
$$

Define

$$
K_{0}=\left\{g: s^{1 / 2} g(s) \text { is nondecreasing for } 0<s<\delta(\delta>0)\right\} .
$$

Let

$$
h^{*}(x)=\sup s^{1 / 2} g(s) \quad \text { for } 0<s \leq x \quad \text { and } \quad g^{*}(x)=x^{-1 / 2} h^{*}(x) \quad(x>0)
$$

where $g$ is any function satisfying (i)-(iii). For $g^{*}$ we define $T^{*}, \varphi^{*}, q^{*}$ and $Q^{*}$ similarly to those corresponding to $g$. Define

$$
\begin{aligned}
J(x) & =\int_{0}^{x} g(s) s^{-1 / 2} d s, \quad J^{*}(x)=\int_{0}^{x} g^{*}(s) s^{-1 / 2} d s, \\
K & =\left\{g: \sup \left(J^{*}(x) / J(x)\right)<\infty \text { for } 0<x \leq \delta\right\} .
\end{aligned}
$$

Of course, $K_{0} \subseteq K$. Moreover, both $K_{0}$ and $K$ contain nondecreasing functions $g$ satisfying (i). We shall also present an example of $g \in K$ which takes the value 0 at some points $x_{n}, n=1,2, \ldots$, such that $x_{n} \rightarrow 0$ as $n \rightarrow \infty$.

If we are interested only in functions $g \in K$, then conditions (1.7) and (1.8) turn out to be equivalent. This is stated in the following theorem.

Theorem 1.2. If $g \in K$, then the condition

$$
\int_{0}^{\delta} \frac{d s}{\left\{s^{1 / 2} \varphi(s)\right\}^{1 / 3}}<\infty \quad(\delta>0)
$$

is necessary and sufficient for the existence of a unique solution $u$ of (1.1) such that $u(x)>0$ for $x>0$.

2. Proofs of theorems. To prove Theorems 1.1 and 1.2 we need three lemmas. In the first one we give an a priori estimate of solutions of (1.2). 
LEMMA 2.1. For any continuous solution $v$ of (1.2) we have

$$
q(x) \leq v(x) \leq Q(x) \quad(x>0) .
$$

Pr o of. Using (1.2) we easily check that $v(x) / x, x>0$, is nondecreasing. Therefore

$$
v(x)=\int_{0}^{x}(x-s) g(s) v(s)^{-1 / 2} d s \geq\left(\frac{v(x)}{x}\right)^{-1 / 2} \varphi(x) \quad(x>0) .
$$

Hence we get the left-hand inequality. Now the right-hand inequality follows from the monotonicity properties of $T$.

The problem of the existence of solutions of (1.2) is considered in the following lemmas.

LEMMA 2.2. There exists at least one continuous solution of (1.2).

Pr o of. We regularize (1.2) as follows:

$$
v_{\varepsilon}(x)=\varepsilon x+\int_{0}^{x}(x-s) g(s) v_{\varepsilon}(s)^{-1 / 2} d s \quad(\varepsilon, x>0),
$$

where $v_{\varepsilon}$ is sought in $C[0, \delta)$. Define

$$
T_{\varepsilon} w(x)=\varepsilon x+\int_{0}^{x}(x-s) g(s) w(s)^{-1 / 2} d s
$$

for any continuous $w:(0, \delta) \rightarrow[0, \infty)$ such that the integral on the righthand side is finite and set

$$
w_{0}(x)=\varepsilon x \quad \text { for } 0<x<\delta \quad \text { and } \quad w_{n}=T_{\varepsilon}^{n} w_{0}, \quad n=1,2, \ldots
$$

The following useful properties of $T_{\varepsilon}$ can easily be obtained:

$$
\begin{gathered}
w_{0} \leq T_{\varepsilon} v \quad \text { for any } v \geq 0 \\
\text { if } 0 \leq v_{1} \leq v_{2}, \text { then } T_{\varepsilon} v_{1} \geq T_{\varepsilon} v_{2} \\
T_{\varepsilon}(c v) \geq c^{-1 / 2} T_{\varepsilon} v \quad \text { for any } c \geq 1 \text { and } v \geq 0 .
\end{gathered}
$$

From (2.3) and (2.4) it follows that

$$
w_{0} \leq w_{2} \leq w_{4} \leq \ldots \leq w_{5} \leq w_{3} \leq w_{1} .
$$

Since

$$
T_{\varepsilon} w_{0}(x)=\varepsilon x+\varepsilon^{-1 / 2} \int_{0}^{x}(x-s) g(s) s^{-1 / 2} d s,
$$

from (iii) it follows that there exists $\delta_{\varepsilon}>0$ such that

$$
w_{0}(x) \leq w_{1}(x) \leq \frac{3}{2} w_{0}(x) \quad \text { for } 0<x<\delta_{\varepsilon} .
$$


Applying (2.5) and using an inductive argument we can see that

$$
1 \leq \frac{w_{2 k+1}(x)}{w_{2 k}(x)} \leq\left(\frac{3}{2}\right)^{(1 / 2)^{2 k}} \quad \text { for } 0<x<\delta_{\varepsilon} \text { and } k=0,1,2, \ldots
$$

From (2.6) and (2.7) we conclude that the sequence $\left\{w_{n}\right\}$ is convergent. Since the function $w(x)=\lim _{n \rightarrow \infty} w_{n}(x), 0<x<\delta_{\varepsilon}$, satisfies $(2.2)$ on $\left(0, \delta_{\varepsilon}\right)$, from its construction it follows that $v_{\varepsilon}(x)=w(x)$ is a unique continuous solution of $(2.2)$ on a $\left(0, \delta_{\varepsilon}\right)$. Now, noting that $v_{\varepsilon}(x) / x$ is nondecreasing, we get the a priori estimates

$$
q(x) \leq v_{\varepsilon}(x) \leq \varepsilon x+Q(x), \quad v_{\varepsilon}^{\prime}(x) \leq \varepsilon+Q^{\prime}(x) \text { for } x>0,
$$

from which we conclude that $v_{\varepsilon}$ can be extended from $\left[0, \delta_{\varepsilon}\right)$ to a unique continuous solution of (2.2) defined on a whole interval independent of $\varepsilon$.

Now, estimate (2.8) allows us to apply the Arzelà-Ascoli theorem. Thus we see that there exists a convergent sequence $\left\{v_{\varepsilon_{n}}\right\}$ of solutions of $(2.2)$, where $\varepsilon_{n} \rightarrow 0$, as $n \rightarrow \infty$. The limit $v(x)=\lim _{n \rightarrow \infty} v_{\varepsilon_{n}}(x), x>0$, is a solution of (1.2), which completes the proof.

A uniqueness result for equation (1.2) is given in the following lemma.

LEMMA 2.3. If there exists a constant $m$ such that

$$
Q(x) \leq m q(x) \quad(0<x \leq \delta),
$$

then the equation (1.2) has exactly one continuous solution $v$.

P r o of. Let $v_{1}, v_{2}$ be two continuous solutions of (1.2). By Lemma 2.1 we have

$$
q(x) \leq v_{1}(x), \quad v_{2}(x) \leq m q(x) \quad(0<x<\delta) .
$$

Therefore there exist constants $c_{1}, c_{2}>0$ such that

$$
c_{1} v_{1}(x) \leq v_{2}(x) \leq c_{2} v_{1}(x) \quad(0 \leq x \leq \delta) .
$$

Note also that for any $c>0$ and for any solution $v$ of (1.2) we have

$$
T^{n}(c v)=c^{(-1 / 2)^{n}} v .
$$

Therefore applying $T^{n}$ to each term of (2.10), by the monotonicity properties of $T$ we easily get $v_{1}=v_{2}$, which completes the proof.

Now we are ready to prove our theorems.

Proof of Theorem 1.1. It is easily seen that Theorem 1.1 is a simple corollary of Lemmas 2.1 and 2.2.

Proof of Theorem 1.2. In view of Theorem 1.1 and Lemma 2.3, to prove Theorem 1.2 it suffices to show that there exists a constant $m$ such that (2.9) is satisfied. 
It follows from the definition of $K$ that there exists a constant $c>0$ such that

$$
c q^{*}(x) \leq q(x) \leq q^{*}(x) \quad \text { for } x \in(0, \delta)
$$

Hence we get

$$
\begin{aligned}
Q(x) & =T q(x) \leq c^{-1 / 2} T q^{*}(x) \\
& \leq c^{-1 / 2} T^{*} q^{*}(x)=c^{-1 / 2} Q^{*}(x) \quad \text { for } x \in(0, \delta) .
\end{aligned}
$$

Now it suffices to note that $g^{*} \in K_{0}$. Therefore, by Lemma $1.2(\mathrm{~b}), Q^{*}(x) \leq$ $\frac{9}{2} q^{*}(x)$ for $x \in(0, \delta)$, which combined with (2.11) and (2.12) gives the required result.

3. Examples. In this section we give two examples of application of the previous results.

EXAMPLE 3.1. Let $g(x)=x^{-1 / 2}(-\ln x)^{-\gamma}, \gamma>0,0<x<\delta$, for some $\delta>0$. If $0<\gamma \leq 1$, then we can easily check that $g$ does not satisfy condition (iii) which, in view of inequality $(2.1)$, is one of the necessary conditions for the existence of nontrivial solutions of (1.1).

If $\gamma>1$, then $g$ belongs to $K_{0}$ and we can easily check that the condition given in Theorem 1.2 is satisfied. Therefore in the case of $\gamma>1$ the problem (1.1) has a unique solution $u$ such that $u(x)>0$ for $0<x<\delta$.

Before giving a second example we consider another subclass of $K$. Namely, let a bounded function $g$ satisfy condition (i). We set

$$
\bar{g}(x)=\sup g(s) \text { for } 0<s \leq x(0<x<\delta),
$$

and we define

$$
G(x)=\int_{0}^{x} g(s) d s, \quad \bar{G}(x)=\int_{0}^{x} \bar{g}(s) d s \quad(0<x<\delta) .
$$

Denote by $K_{1}$ the class of bounded functions $g$ satisfying (i) and such that

$$
\sup (\bar{G}(x) / G(x))<\infty \quad \text { for } x \in(0, \delta) .
$$

Remark 3.1. $K_{1} \subseteq K$.

Pro of. Let $g \in K_{1}$. Since $g$ is bounded, an integration by parts shows that

$$
\int_{0}^{x} g(s) s^{-1 / 2} d s=x^{-1 / 2} G(x)+\frac{1}{2} \int_{0}^{x} G(s) s^{-3 / 2} d s .
$$

Using the same formula for $\bar{g}$ we obtain

$$
\int_{0}^{x} \bar{g}(s) s^{-1 / 2} d s \leq c \int_{0}^{x} g(s) s^{-1 / 2} d s \quad \text { for } x \in(0, \delta),
$$


where $c>0$ is some constant. Hence and by the fact that $g^{*} \leq \bar{g}$ on $(0, \delta)$ it follows that $g \in K$, which ends the proof.

In the case of $g \in K_{1}$ the following theorem gives a necessary and sufficient condition for the existence of nontrivial solutions of (1.1).

THEOREM 3.1. Let $g \in K_{1}$. Then (1.1) has a unique solution $u$ such that $u(x)>0$ for $x>0$ if and only if

$$
\int_{0}^{\delta}\left(\frac{s}{\bar{g}(s)}\right)^{1 / 3} \frac{d s}{s}<\infty \quad(\delta>0) .
$$

Proof. Since $\bar{g}$ is nondecreasing, we get

$$
2^{-1} \bar{g}(x / 2) x^{1 / 2} \leq \int_{0}^{x} \bar{g}(s) s^{-1 / 2} d s \leq 2 \bar{g}(x) x^{1 / 2} \quad(0<x<\delta) .
$$

Therefore the required result follows from Theorem 1.2 by applying (3.1).

EXAMPLE 3.2. Let $x_{k}=(2 k+1)^{-1} \pi^{-1}, y_{k}=(2 k \pi)^{-1}, a_{k}=$ $(2 k+5 / 6)^{-1} \pi^{-1}, b_{k}=(2 k+1 / 6)^{-1} \pi^{-1}$ for any integer $k \geq k_{0}$, where $k_{0}$ is a fixed integer number such that $y_{k_{0}} \in(0, \delta)$. We define

$$
g(x)= \begin{cases}\sin 1 / x & \text { for } x_{k} \leq x \leq y_{k} \\ 0 & \text { for } y_{k+1}<x<x_{k}\left(k>k_{0}\right)\end{cases}
$$

and

$$
g_{1}(x)= \begin{cases}1 / 2 & \text { for } a_{k} \leq x \leq b_{k}, \\ 0 & \text { for } b_{k+1}<x<a_{k}\left(k>k_{0}\right) .\end{cases}
$$

Thus we have $g_{1}(x) \leq g(x) \leq \bar{g}(x)=1$ for $x \in(0, \delta)$. Set

$$
\begin{gathered}
G_{1}(x)=\int_{0}^{x} g_{1}(s) d s, \quad \bar{G}(x)=\int_{0}^{x} \bar{g}(s) d s=x \quad \text { for } x \in(0, \delta), \\
r_{k}=\sum_{l=k}^{\infty}\left(b_{l}-a_{l}\right) \quad \text { for } k>k_{0} .
\end{gathered}
$$

For $x \in\left(a_{k+1}, a_{k}\right)\left(k>k_{0}\right)$ we get

$$
\frac{\bar{G}(x)}{G(x)} \leq \frac{x}{G_{1}(x)} \leq 2 \frac{x}{r_{k+1}} \leq 2 \frac{a_{k}}{r_{k+1}}=2 \frac{a_{k+1}}{r_{k+1}} \frac{a_{k}}{a_{k+1}} .
$$

Of course, $a_{k} / a_{k+1}<c$ for some constant $c$ and $k>k_{0}$. Since

$$
r_{k}=\pi^{-1} \sum_{l=k}^{\infty}(2 l+5 / 6)^{-1}(2 l+1 / 6)^{-1},
$$

we can compare $r_{k}$ with $\int_{k}^{\infty} s^{-2} d s$. As a result, $a_{k} / r_{k}<c$ for some constant $c$ and $k>k_{0}$. Now, by (3.3) we conclude that $g \in K_{1}$. Applying Theorem 3.1 we see that the problem (1.1) with $g$ defined in (3.2) has a nontrivial solution. 
Acknowledgements. This paper was written while the author was visiting Freie Universität Berlin in November 1992 as a guest to the research group "Regularization". The author wishes to thank Prof. R. Gorenflo for helpful discussions on convolution type integral equations.

\section{References}

[1] P. J. Bushell and W. Okrasiński, Uniqueness of solutions for a class of nonlinear Volterra integral equations with convolution kernel, Math. Proc. Cambridge Philos. Soc. 106 (1989), 547-552.

[2] G. Gripenberg, Unique solutions of some Volterra integral equations, Math. Scand. 48 (1981), 59-67.

[3] W. Mydlarczyk, The existence of nontrivial solutions of Volterra equations, ibid. 68 (1991), 83-88.

[4] W. Okrasiński, Nontrivial solutions to nonlinear Volterra integral equations, SIAM J. Math. Anal. 22 (1991), 1007-1015.

[5] -, Nontrivial solutions for a class of nonlinear Volterra equations with convolution kernel, J. Integral Equations Appl. 3 (1991), 399-409.

INSTITUTE OF MATHEMATICS

UNIVERSITY OF WROCEAW

PL. GRUNWALDZKI $2 / 4$

50-384 WROCŁAW, POLAND

Reçu par la Rédaction le 25.1.1993

Révisé le 3.6.1993 